\title{
ALGEBRAIC FIELD THEORY ON CURVED MANIFOLDS
}

\author{
MARTIN OLESEN
}

\begin{abstract}
In this paper we set up an algebraic framework for the study of quantum field theory in a class of manifolds, which includes Minkowski space and the Kruskal spacetime. The formalism provides a unifying framework for studying problems of Bisognano-Wichmann type, e.g., Hawking radiation in black hole geometries.

Analogously to flat spacetime, we establish a correspondence between isometries of certain wedge domains of spacetime and the modular structure of the local algebras. Under an ergodic hypothesis, the wedge algebras are shown to be type III factors as expected, and we derive a result concerning factorization of the equilibrium state. This result generalizes a similar one obtained by Sewell in [Ann. Phys. 141 (1982), 201-224].

Finally an example of a quantum field theory satisfying the basic axioms is constructed. The local algebras are field algebras of bosonic free field solutions to the Klein-Gordon equation twisted through a PCT-like conjugation, and we show that this model realizes the abstract properties developed on the axiomatic basis.
\end{abstract}

\section{INTRODUCTION}

Hawking [Ha] in 1975 predicted that particle creation in the vicinity of a black hole could lead to evaporation of it, and this led several researchers to formulate rigorous quantum field theories in black hole metrics, e.g., [Da], [U], [Fu], [Se], [Ka] and references therein. It was discovered that thermalization of states in quantum field theory by gravitational forces is a general phenomenon in nonflat metrics.

Sewell [Se] observed the connection of these results with the BisognanoWichmann formalism in Minkowski space and constructed an axiomatic Wightmann field theory in a certain class of curved manifolds. In this setting, he was able to give a rigorous proof of the thermalization properties and to prove analogues of the Reeh-Schlieder, Bisognano-Wichmann, and PCT-theorems.

However, in Sewell's axiomatics, ad hoc axioms, on the analytic behavior of the fields along certain light cones, had to be introduced. It seems desirable to construct a simpler framework for deriving structural results, in the setting of [Se], [Ka] and others.

Received by the editors November 11, 1992 and, in revised form, November 1, 1993; originally communicated to the Proceedings of the AMS by Palle E. T. Jorgensen.

1991 Mathematics Subject Classification. Primary 81E20, 81E05, 46L60.

Key words and phrases. Quantum field theory, space-time manifolds, von Neumann algebras, modular theory.

The work in this paper was supported by a grant from the Carlsberg Foundation. 
The algebraic formulation of quantum field theory (see $[\mathrm{H}],[\mathrm{HK}],[\mathrm{Ho}])$ provides a conceptually simple general framework, with all the assumptions reflecting fundamental physical ideas. In this paper we propose a set of axioms specifically tailored to generalize the usual Haag-Kastler axioms to the class of spacetimes studied by Sewell [Se]. This class consists of spacetimes of the form $X=\mathbb{R}^{2} \times Y$, with a metric given by the line element

$$
d s^{2}=A\left(t^{2}-w^{2}, y\right)\left(d t^{2}-d w^{2}\right)-B\left(t^{2}-w^{2}, y\right) d \sigma^{2}(y)
$$

where $A$ and $B$ are smooth, strictly positive functions on $X, d \sigma^{2}(y)$ is a positive definite metric on $Y$, and $(t, w)$ are canonical coordinates in $\mathbb{R}^{2}$. Notice that $X$ is globally hyperbolic. We will be especially interested in studying the two spacetime regions $X^{ \pm}$, given by

$$
X^{ \pm}=W^{ \pm} \times Y
$$

where $W^{ \pm}$is the right (resp., left) wedge in $\mathbb{R}^{2}$, given by $w>|t|$ (resp., $-w>|t|)$.

Apart from the Minkowski space, with $X^{ \pm}$the Rindler wedges, this class contains several interesting black hole backgrounds. For example, letting $Y$ be the two-sphere $S^{2}$, with $d \sigma^{2}=d \theta^{2}+\sin ^{2} \theta d \phi^{2} ; A=\exp (-r / 2 m) 16 m^{2} / r$; and $B=r^{2}$, with $r$ determined implicitly from $-(r-2 m) \exp (r / 2 m)=t^{2}-w^{2}$, we obtain the Kruskal manifold, with $X^{ \pm}$the exterior and interior Schwarzchild spacetimes respectively. Here $m$ is a parameter, which can be thought of as the total mass of the black hole.

The paper consists of three sections. In the first we list and discuss our quantum axioms.

In section II we derive our structural theorems. As mentioned above, the thermalization effect of gravitation on quantum fields is reminiscent of the Bisognano-Wichmann formalism [BW]. The natural objects of study are therefore the local algebras $\mathfrak{R}\left(X^{ \pm}\right)$of the generalized wedges $X^{ \pm}$. These regions carry one-parameter actions of isometries consisting of generalized Lorentz boosts $L$. In general it is not possible to assume that the selfadjoint generator in the unitary representation of $L$ is positive, but if there exist a bicyclic vector for the wedge-algebras $\mathfrak{R}\left(X^{ \pm}\right)$, modular theory gives alternative analytic information.

Thus, under the presence of a bicyclic, invariant vector $\Omega$ for $\mathfrak{R}\left(X^{+}\right)$, we give in Theorem 3 an invariance condition of the unitary group of generalized Lorentz boosts, characterizing the case in which this group (up to a rescaling) equals the modular group of the pair $\left(\mathfrak{R}\left(X^{ \pm}\right), \Omega\right)$. Moreover, a slightly stronger condition proves to be equivalent to duality for the wedge-algebras:

$$
\mathfrak{R}\left(X^{+}\right)^{\prime}=\mathfrak{R}\left(X^{-}\right) .
$$

These results are direct analogues of the Bisognano-Wichmann theorem [BW] and generalize similar results obtained by Rigotti in [R].

We then study the consequences of an ergodic hypothesis, to the effect that the vector state $\omega_{\Omega}$ is the unique invariant state on $\mathfrak{R}\left(X^{ \pm}\right)$. Under this hypothesis, we are able to prove in Theorem 4 that the wedge-algebras are type III factors. Moreover, in Theorem 5 we prove that the state $\omega_{\Omega}$ has the following product property $\omega_{\Omega}\left(A \sigma_{-i / 2} B\right)=\omega_{\Omega}(A) \omega_{\Omega}(B)$, where $A \in \mathfrak{R}\left(X^{+}\right), B \in \mathfrak{R}\left(X^{-}\right)$, and $\sigma_{t}$ is the modular group for the pair $\left(\mathfrak{R}\left(X^{+}\right), \Omega\right)$. This generalizes a result 
of Sewell [Se, Theorem 10], who proved that $\omega_{\Omega}(A B)=\omega_{\Omega}(A) \omega_{\Omega}(B)$ in the case where $\omega_{\Omega}$ is a ground state, i.e., where the unitary group of generalized Lorentz boosts have a positive generator. In Sewell's case the interpretation of the result is that the observables in $X^{+}$and $X^{-}$are mutually uncorrelated in the state $\omega_{\Omega}$. In the case where $X$ is the Kruskal manifold and $X^{ \pm}$the exterior and interior Schwartzschild spacetimes respectively, this implies that the event horizon around a Schwartzschild black hole acts as a physical barrier that prevents correlation between the observables on the two sides of the event horizon. It would be interesting to get a similar interpretation of the generalized result, but what that should be is not clear.

Finally, in section III, we construct a model quantum field theory satisfying the basic axioms of section I. The construction is similar to the one in [D], however, we show that the model satisfies all the abstract properties proved in section II.

\section{THE QUANTUM AXIOMS}

The isometry group of $X$ contains a one-parameter group $L(\tau), \tau \in \mathbb{R}$, which acts as the identity on the $Y$ factor and as Lorentz boosts around the $w$-axis on the $\mathbb{R}^{2}$ factor. $L(\tau)$ induces a timelike Killing vector field in the wedgelike regions $X^{ \pm}$. It is therefore possible to find a time coordinate in these regions. By carrying out the coordinate transformation $w=\xi \cosh \tau, t=\xi \sinh \tau$ with $\xi>0(<0)$ in $X^{+}\left(X^{-}\right)$we get

$$
d s^{2}=A\left(-\xi^{2}, y\right)\left(\xi^{2} d \tau^{2}-d \xi^{2}\right)-B\left(-\xi^{2}, y\right) d \sigma^{2}(y)
$$

and we see that $\tau$ is a time coordinate in $X^{ \pm}$. Furthermore, each $L\left(\tau_{0}\right)$ induces on $X^{ \pm}$the time translation $\tau \rightarrow \tau+\tau_{0}$.

We will now formulate the axioms for a quantum field theory in $X$. The basic axioms are the following five, which are analogous to the Minkowski space axioms [Ho]:

(I) There exist a seperable Hilbert space $\mathscr{H}$ and a net $\mathscr{O} \rightarrow \mathfrak{R}(\mathscr{O})$ of von Neumann algebras on $\mathscr{H}$, indexed by precompact open $\mathscr{O} \subset X$ and directed by inclusion. The assignment $\mathscr{O} \rightarrow \mathfrak{R}(\mathscr{O})$ is called a net of local observables. The $W^{*}$-inductive limit $\mathfrak{R}$ of the net of local observables is called the global algebra. We remark that we assume that all the local algebras have a common unit.

(II) The net of local observables is strongly additive, i.e., if $\mathscr{O}=\bigcup_{i \in J} \mathscr{O}_{i}$ for $\mathscr{O}, \mathscr{O}_{i} \in B(X)$, then $\mathfrak{R}(\mathscr{O})=\left(\bigcup_{i \in J} \mathfrak{R}\left(\mathscr{O}_{i}\right)\right)^{\prime \prime}$.

(III) If $S$ is a spacelike Cauchy surface in $X$, then, for any open neighborhood $\mathscr{O}$ of $S$, we have $\mathfrak{R}(\mathscr{O})=\mathfrak{R}$. This "time-slice axiom" is an expression of the causal propagation properties of the theory.

(IV) The net of local observables satisfies locality, that is, if $\mathscr{O}_{1} \subset \mathscr{O}_{2}^{\prime}$, then $\mathfrak{R}\left(\mathscr{O}_{1}\right) \subset \mathfrak{R}\left(\mathscr{O}_{2}\right)^{\prime}$. Here $\mathscr{O}_{2}^{\prime}$ means the spacelike complement of $\mathscr{O}_{2}$, i.e. the set of points in $X$, which cannot be joined to $\mathscr{O}_{2}$ with a smooth, nowhere spacelike curve.

(V) There exist a strongly continuous, unitary representation, also denoted by $L$, of $L$, on $\mathscr{H}$, satisfying

$$
L(\tau) \mathfrak{R}(\mathscr{O}) L(\tau)^{-1}=\mathfrak{R}(L(\tau) \mathscr{O}), \quad \tau \in \mathbb{R}, \mathscr{O} \in B\left(X^{+} \cup X^{-}\right) .
$$


We remark that because of the strong additivity it is consistent to define $\mathfrak{R}(\mathscr{O})=\left\{A \in \mathfrak{R}\left(\mathscr{O}_{i}\right) \mid \mathscr{O}_{i} \in B(X), \mathscr{O}_{i} \subset \mathscr{O}\right\}^{\prime \prime}$ for unbounded domains $\mathscr{O}$. Moreover, as $L(\tau)$ does not act isometrically on $X \backslash\left(\overline{X^{+} \cup X^{-}}\right)$, we cannot assume it satisfies (2) on this subspace.

An attempt to generalize the usual Haag-Kastler axioms to a nonflat spacetime runs into the problem that the isometry group is not in general sufficiently big. Indeed, in the Minkowski space all the really powerful results stem from the fact that the isometry group of Poincare transformations acts transitively on Minkowski space and satisfy the spectrum condition. In our case, we have the one-parameter isometry group $L$ which does not act transitively on $X$, or even on $X^{ \pm}$. Moreover we cannot demand a spectrum condition on $L$ if we want the framework to be a genuine generalization of the Haag-Kastler axioms. Indeed, in Minkowski space, it is well known that the selfadjoint generator $H$ of $L(\tau)$ has the entire real line as spectrum; see, e.g., [F]. As the existence of a positively represented and transitive isometry group is the key ingredient in proving the Reeh-Schlieder theorem, on the existence of cyclic and separating vectors for the local algebras, we have to do without this theorem. In this paper we will be concerned with the structure of the wedge algebras $\mathfrak{R}\left(X^{ \pm}\right)$, and we propose to introduce the following axiom to give us bicyclic vectors.

(VI) There exist common cyclic vectors for the two algebras $\mathfrak{R}\left(X^{+}\right)$and $\mathfrak{R}\left(X^{-}\right)$.

From locality we have $\mathfrak{R}\left(X^{ \pm}\right) \subset \mathfrak{R}\left(X^{\mp}\right)^{\prime}$, so we see that this axiom actually garantees the existence of common bicyclic vectors for the wedge-algebras.

\section{THE STRUCTURE OF THE WEDGE-ALGEBRAS}

Let $\omega$ be a normal state of $\mathfrak{R}$, and assume that $\omega$ is invariant with respect to $L$.

From axiom (VI) it follows that there exists separating vectors for $\mathfrak{R}\left(X^{+}\right)$. $\omega$ is therefore a vector state [KR, Theorem 7.2.3], so there exists a unit vector $\Omega \in \mathscr{H}$, such that $\omega=\left.\omega_{\Omega}\right|_{\mathscr{R}}$ (here $\omega_{\Omega}$ means the state $\omega_{\Omega}(A)=\langle\Omega, A \Omega\rangle$ ). Also we remark that it is possible to adjust the unitary implementation of $L$ by a unitary one-parameter group from $\mathfrak{R}\left(X^{+} \cup X^{-}\right)^{\prime}$ such that $\Omega$ is $L$-invariant, and we will always assume this done. In our later applications $\mathfrak{R}\left(X^{+}\right)$and $\mathfrak{R}\left(X^{-}\right)$will be commuting factors, so $L$ is unique.

With such $\omega, \Omega$, Tomita's Theorem [KR, Theorem 9.2.9] implies the existence of a conjugate linear isometry $J: \mathscr{H} \rightarrow \mathscr{H}$ and a densely defined, positive, selfadjoint operator $\Delta$ in $\mathscr{H}$, such that

$$
\begin{aligned}
& J \Delta^{\frac{1}{2}} A \Omega=A^{*} \Omega \text { for all } A \in \mathfrak{R}\left(X^{+}\right), \\
& J \mathfrak{R}\left(X^{+}\right) J=\mathfrak{R}\left(X^{+}\right)^{\prime}, \\
& \Delta^{i \tau} \mathfrak{R}\left(X^{+}\right) \Delta^{-i \tau}=\mathfrak{R}\left(X^{+}\right), \\
& J \Omega=\Delta \Omega=\Omega, \\
& J^{*}=J, \quad J \Delta J=\Delta^{-1},
\end{aligned}
$$

and a strongly continuous unitary one-parameter group $\sigma_{\tau}$ of automorphisms of $\mathfrak{R}\left(X^{+}\right)$given by

$$
\sigma_{\tau}(A)=\Delta^{i \tau} A \Delta^{-i \tau} .
$$

The pair $(J, \Delta)$ is called a modular pair, and it depends on $\Omega$. 
Our first aim is to show that, under a natural invariance condition on the group representation $L(\tau)$, the automorphism group $\operatorname{ad} L(2 \pi \tau)$, given by ad $L(\cdot)(A):=L(\cdot) A L(\cdot)^{*}$, coincides with the modular group $\sigma_{\tau}$ on $\mathfrak{R}\left(X^{+}\right)$. This leads to the interpretation of $\omega$ as a temperature state in $X^{+}$(and in $X^{-}$) of temperature $T=\hbar / 2 \pi k$, with respect to the dynamical group $L(\tau)$, where $k$ is Boltzmann's constant [HHW]. An observer, travelling along the worldlines $\xi=$ constant, will experience the proper time $c^{-1}\left(A\left(-\xi^{2}, y\right)\right)^{\frac{1}{2}} \tau$. In cases where the local algebras are field algebras of some quantum field (see [Ho, Chapter 3]), the observer will see the field as having a temperature of $T=\hbar c / 2 \pi k\left(A\left(-\xi^{2}, y\right)\right)^{\frac{1}{2}}$. This yields another formal proof of the fact that a gravitational field can thermalize a quantum field, something that was suggested heuristically by Hawking in [Ha].

The key to this result is to study the complexification of the group $L(\tau)$. Consider the embedding

$$
X=\mathbb{R}^{2} \times Y \hookrightarrow \mathbb{C}^{2} \times Y
$$

with $X$ naturally identified with the set of points $\left(z, z^{\prime}, y\right)$ with real $z$ and $z^{\prime}$. On $\mathbb{C}^{2} \times Y$, the complexification $L(z), z \in \mathbb{C}$, of $L(\tau)$ acts by complex Lorentz boosts on the $\mathbb{C}^{2}$ factor.

The transformation $L(i \pi)$ has particular interest, since it induces the partial inversion $(t, w, y) \rightarrow(-t,-w, y)$ on $X$.

If $d \mu(s)$ denotes the spectral measure of a unitary group $U(\tau)=e^{i \tau H}$, we can always extend $U$ to a representation of $\mathbb{C}$ by putting

$$
U(z)=\int_{\mathbb{R}} e^{i z s} d \mu(s) \text { for all } z \in \mathbb{C},
$$

although the operators $U(z)$ are then in general unbounded. The following lemma gives an explicit description of a common core for them.

Lemma 1. Let $\mathfrak{R}$ be a von Neumann algebra on a Hilbert space $\mathscr{H}, \Omega \in \mathscr{H}$ a cyclic vector for $\mathfrak{R}$; and $U(\tau), \tau \in \mathbb{R}$, a strongly continuous unitary group on $\mathscr{H}$ implementing automorphisms of $\mathfrak{R}$. If $\Omega$ is invariant under $U(\tau)$ for all $\tau \in \mathbb{R}$, then $\mathfrak{R} \Omega$, is a core for $U(z)$ for all $z \in \mathbb{C}$.

Proof. We show that $\mathscr{D}(\tau) \equiv(1+U(i \tau))^{-1} \mathscr{H}, \tau \in \mathbb{R}$ is a core for all the operators $U(z)$ with $\operatorname{Im} z=\tau$. As the domain of $U(z)$ only depends on $\operatorname{Im} z$, it is enough to consider $U(i \tau)$. Since $\operatorname{spec}(U(i \tau)) \subset \mathbb{R}^{+}$, the operator $(1+U(i \tau))$ is invertible, so $\mathscr{D}(\tau)$ is well defined. We show that $(U(i \tau), \mathscr{D}(\tau))$ is closed. This implies that it is selfadjoint and, as the adjoint have dense domain, that $\mathscr{D}(\tau)$ is a core for $U(i \tau)$. Let $x_{n} \rightarrow 0$ from $\mathscr{D}(\tau)$, and assume that $U(i \tau) x_{n} \rightarrow y$. We have $(1+U(i \tau)) x_{n}=y_{n} \rightarrow 0+y=y$. Since $(1+U(i \tau))^{-1}$ is bounded and $y_{n} \rightarrow y$, we have $x_{n}=(1+U(i \tau))^{-1} y_{n} \rightarrow$ $(1+U(i \tau))^{-1} y=0$, so $y \in \operatorname{ker}(1+U(i \tau))^{-1}$. But as $(1+U(i \tau))^{-1}$ is a bijection of $\mathscr{H}$ on $\mathscr{D}(\tau)$, we have $y=0$. We conclude that $(U(i \tau), \mathscr{D}(\tau))$ is closed.

Now define $C_{f}=\operatorname{span}\left\{f(H) \Re \Omega \mid f \in C_{c}^{\infty}(\mathbb{R})\right\}$, where $H$ is the infinitesimal generator of $U(\tau)$. Then $(1+U(i \tau))^{-1}=\left(1+e^{\tau H}\right)^{-1}$. We can approximate the real function $f(x)=\left(1+e^{\tau x}\right)^{-1}$ with functions from $C_{c}^{\infty}(\mathbb{R})$ in the uniform topology. Hence, the spectral theorem gives us a net of the form $f_{\mu}(H)$ 
converging strongly to $(1+U(i \tau))^{-1}$. It then follows that $C_{f}$ is dense in all $\mathscr{D}(\tau)$.

Now observing that if $f \in C_{c}^{\infty}(\mathbb{R})$ and $A \in \mathfrak{R}$, then the operator

$$
A_{f}=\int_{\mathbb{R}} \hat{f}(\tau) U(\tau) A U(\tau)^{-1} d \tau
$$

belongs to $\mathfrak{R}$, and $A_{f} \Omega=f(H) A \Omega$. As $\Omega$ is cyclic for $\mathfrak{R}, \mathfrak{R} \Omega$ is a dense subspace of $\mathscr{H}$ and it follows from the above considerations that $\Re \Omega$ is a core for $U(i \tau)$.

The key to the proof that $L(2 \pi \tau)$ implements the modular group of the dynamical system $\left(\mathfrak{R}\left(X^{ \pm}\right), \Omega\right)$, with $\Omega$ bicyclic for $\mathfrak{R}\left(X^{+}\right)$, is the observation that the invariance properties of the core $\Re\left(X^{+}\right) \Omega$ of $L(i \pi)$ determines $L$. This is the result of the next lemma, which proves a result of [R] under slightly weaker assumptions.

Lemma 2. Let $\mathfrak{R}$ be a von Neumann algebra on a Hilbert space $\mathscr{H}$, with a bicyclic vector $\Omega \in \mathscr{H}$. Let $U(t), t \in \mathbb{R}$, be a strongly continuous unitary group such that $U(t) \Omega=\Omega$ and $U(t) \mathfrak{R} U(t)^{*}=\mathfrak{R}$, for all $t \in \mathbb{R}$. If the subspace $\mathfrak{R} \Omega$ is invariant under $U(i \pi) \quad\left(=e^{\pi K}\right.$ with $K$ the infinitesimal generator of $\left.U(t)\right)$, then $U(t)=1$ for all $t \in \mathbb{R}$.

Proof. Assume that $A \in \mathfrak{R}$ is selfadjoint. If $U(i \pi) \mathfrak{R} \Omega \subset \mathfrak{R} \Omega$, there is a $C \in \mathfrak{R}$ such that $U(i \pi) A \Omega=C \Omega$.

Let $S \mapsto E(S), S \subset \operatorname{spec}(K)$ a Borel subset, be the projection-valued spectral measure of $K$. Then for any $x \in \mathscr{H}$ and $y \in \mathfrak{R} \Omega$ we have

$$
\left\langle x, e^{\pi K} y\right\rangle=\int e^{\pi \lambda} d \mu_{x, y}(\lambda)
$$

where $\mu_{x, y}(S)=\langle x, E(S) y\rangle$. In particular, putting $x=\Omega, y=A \Omega$ we obtain $\mu_{\Omega, A \Omega}(S)=\langle A E(S) \Omega, \Omega\rangle$ for any Borel set $S \subset \operatorname{spec}(K)$. Thus $d \mu_{\Omega, A \Omega}(\lambda)$ is a real-valued measure, and

$$
\langle\Omega, U(i \pi) A \Omega\rangle=\langle\Omega, C \Omega\rangle \in \mathbb{R} .
$$

So $\left\langle\Omega,\left(C-C^{*}\right) \Omega\right\rangle=0$, and, as $\Omega$ is seperable, $C-C^{*}=0$, so $C$ is selfadjoint. Thus the double cone $\mathfrak{R}^{\mathrm{sa}} \Omega$ in $\mathscr{H}$ is invariant under $U(i \pi)$. Here $\mathfrak{R}^{\text {sa }}$ means the selfadjoint elements of $\mathfrak{R}$.

Now assume $A \in \mathfrak{R}^{\prime \text { sa }}$ and $B \in \mathfrak{R}^{\text {sa }}$. From Lemma $1, \mathfrak{R} \Omega$ is a core for $U(z)$ for all $z \in \mathbb{C}$. So we can define the function

$$
f(z)=\langle A \Omega, U(z) B \Omega\rangle, \quad 0 \leq \operatorname{Im} z \leq \pi,
$$

which is then continuous and bounded on the strip $0 \leq \operatorname{Im} z \leq \pi$ and analytic in the interior of this strip.

From this point on the argument is identical to [R, Lemma 2]. Indeed, noticing that $f$ is real on the real axis and on the line $\operatorname{Im} z=\pi$, so $\mathrm{f}$ extends by analytic reflection to a bounded entire function which is then constant. So

$$
\langle A \Omega, U(t) B \Omega\rangle=\langle A \Omega, B \Omega\rangle
$$

for all $t \in \mathbb{R}$. As $\Omega$ is cyclic and separating for $\mathfrak{R}$, this implies that $U(t)=$ I.

These lemmas lead to the first important result, whose proof is analogous to [R, Theorem 4]. 
Theorem 3. Let a field theory in $X$ satisfy axioms (I)-(VI), and let $\omega$ be an invariant, normal state on the global algebra $\mathfrak{R}$ implemented by an L-invariant bicyclic vector $\Omega$. Then $L(2 \pi \tau), \tau \in \mathbb{R}$, is the modular group of the state $\omega$ on $\mathfrak{R}\left(X^{+}\right)$if and only if

$$
L(i \pi) \mathfrak{R}\left(X^{+}\right) \Omega \subset \mathfrak{R}\left(X^{+}\right)^{\prime} \Omega .
$$

Moreover, the duality (1) is satisfied, and the modular groups of $\mathfrak{R}\left(X^{+}\right)$and $\mathfrak{R}\left(X^{-}\right)$are each other's inverses if and only if

$$
L(i \pi) \mathfrak{R}\left(X^{+}\right) \Omega \subset \mathfrak{R}\left(X^{-}\right) \Omega .
$$

Sketch of proof. We remark first that since $\mathfrak{R}\left(X^{+}\right) \Omega$ is a core for $L(i \pi)$ from Lemma 1 , the two conditions on this operator make sense.

Assume first (3), and consider the unitary group

$$
U(\tau)=\Delta^{-i \tau / 2 \pi} \exp (i \tau H)
$$

Then $\omega$ and $\mathfrak{R}^{+}$are $U$-invariant, and, from Lemma $1, \mathfrak{R}\left(X^{+}\right) \Omega \subset \mathscr{D}(L(i \pi))$. So $U(i \pi)$ satisfies

$$
U(i \pi) \mathfrak{R}\left(X^{+}\right) \Omega \subset \mathfrak{R}\left(X^{+}\right) \Omega .
$$

From Lemma 2, $\exp (i \tau H)=\Delta^{i \tau / 2 \pi}$.

Now, assume (4). The duality would follow from Rieffel-van Deale's commutation theorem [RvD] if, for $\xi \in \mathscr{H}$ and all $A \in \mathfrak{R}\left(X^{+}\right)^{\text {sa }}$ and $B \in \mathfrak{R}\left(X^{-}\right)^{\text {sa }}$ we knew that $\operatorname{Im}\langle\xi, A \Omega\rangle=\operatorname{Re}\langle\xi, B \Omega\rangle=0$ implies $\xi=0$. But this follows from the application of complex function techniques analogous to those in Lemma 2.

In Minkowski space it follows from the basic postulates of the theory of local observables that there exists a unique vacuum state. Generalizing this, Sewell in [Se] studied some consequences of assuming the existence of a unique ground state in $X$. However, in general there does not exist ground states for $\left(\mathfrak{R}\left(X^{ \pm}\right), L(\tau)\right)$ and we must assume that the spectrum of the infinitesimal generator $H$ of $L(\tau)$ is two-sided. It is therefore interesting to assume that there exist a unique $L$-invariant state on $\mathfrak{R}\left(X^{ \pm}\right)$but dropping the positivity assumption on $H$. Theorem 5 below shows that Theorem 10 of [Se] can be generalized to this situation. First, however, we show that the above ergodic hypothesis implies that the wedge algebras are type III factors.

Theorem 4. Let a field theory in $X$ satisfy axioms (I)-(VI), (3) and (4) for a bicyclic vector $\Omega$ for the wedge algebras $\mathfrak{R}\left(X^{ \pm}\right)$. If $\omega_{\Omega}$ is the only temperature state on $\mathfrak{R}\left(X^{+}\right)$, then the wedge algebras are type III factors.

Proof. Let us first show that $\mathfrak{R}\left(X^{+}\right)$is a factor under the hypothesis of the theorem. From Theorem 3, $L(\tau)$ implements the modular group of $\left(\mathfrak{R}\left(X^{+}\right), \Omega\right)$. Thus, if we denote the corresponding modular pair by $(\Delta, J)$,

$$
\Delta^{ \pm \frac{1}{2}} B \Omega=J B^{*} \Omega \text { for all } B \in \mathfrak{R}^{ \pm}
$$

If $A \in \mathfrak{R}\left(X^{+}\right) \cap \mathfrak{R}\left(X^{-}\right)$, this implies $A \Omega \in \mathscr{D}\left(\Delta^{\frac{1}{2}}\right) \cap \mathscr{D}\left(\Delta^{-\frac{1}{2}}\right)$, and we get $\Delta^{\frac{1}{2}} A \Omega=\Delta^{-\frac{1}{2}} A \Omega$ or $\Delta A \Omega=A \Omega$. If $H$ is the selfadjoint infinitesimal generator of $L(\tau)$, this equation implies that $A \Omega$ is an eigenvector for $H$ with eigenvalue 0 and hence that $A \Omega$ is invariant under $L(\tau)$. But as $\omega_{\Omega}$ was the unique 
invariant state, $A \Omega=\lambda \Omega$, and, as $\Omega$ is seperating for $\mathfrak{R}\left(X^{+}\right)$, we get $A=\lambda I$. Hence $\mathfrak{R}\left(X^{+}\right)$is a factor.

To show that this factor is of type III, we remark that Størmer in [St] proved that in our setting, either $\mathfrak{R}\left(X^{+}\right)$is of type III or $\omega_{\Omega}$ is a trace state. Pedersen and Takesaki in [PT, Theorem 3.6] characterized the fixpoint algebra $\mathfrak{R}\left(X^{+}\right)^{\Delta}$ of the action of the modular group on $\mathfrak{R}\left(X^{+}\right)$as

$$
\mathfrak{R}\left(X^{+}\right)^{\Delta}=\left\{A \in \mathfrak{R}\left(X^{+}\right) \mid\langle\Omega, A B \Omega\rangle=\langle\Omega, B A \Omega\rangle, \forall B \in \mathfrak{R}\left(X^{+}\right)\right\} .
$$

If $\omega_{\Omega}$ is a trace state, it follows that $\mathfrak{R}\left(X^{+}\right)=\mathfrak{R}\left(X^{+}\right)^{\Delta}$ and as $\Omega$ is cyclic for $\mathfrak{R}\left(X^{+}\right)$that $\Delta^{i t}=1$. But this contradicts the ergodicity assumption. Hence $\mathfrak{R}\left(X^{+}\right)$is of type III. As a von Neumann algebra is a factor of type III if and only this also holds for the commutant we also obtain that $\mathfrak{R}\left(X^{-}\right)$is a type III factor.

It is interesting that, in contrast to the situation in Minkowski space [F], our framework does not allow us to conclude that the wedge algebras are of type III $_{1}$. In fact, it is possible to construct examples of field theories satisfying all the axioms (I)-(VI) and whose wedge algebras are of type III $_{\lambda}$ for any $\lambda \in] 0,1[$. As a rule this will be a sign that the spacetime lacks asymptotic regions in some direction, e.g., if it has a compact factor, see [O].

The following result shows that the state $\omega_{\Omega}$ factorizes, up to an action of $\sigma_{i / 2}$.

Theorem 5. Let a field theory in $X$ satisfy axioms (I)-(VI) and (3) for a bicyclic vector $\Omega$ for the wedge algebras $\mathfrak{R}\left(X^{ \pm}\right)$, and let $\sigma_{t}$ be the modular group for $\left(\mathfrak{R}\left(X^{+}\right), \Omega\right)$. If $\omega_{\Omega}$ is the only temperature state on $\mathfrak{R}\left(X^{+}\right)$, then this state has the following product property:

For all elements $A \in \mathfrak{R}\left(X^{+}\right)$and $B \in \mathfrak{R}\left(X^{-}\right)$which are entire analytic for the modular group we have

$$
\omega_{\Omega}\left(\sigma_{-i / 2}(A) B\right)=\omega_{\Omega}\left(A \sigma_{i / 2}(B)\right)=\omega_{\Omega}(A) \omega_{\Omega}(B) .
$$

Proof. Note that $\omega_{\Omega}\left(A \sigma_{-i / 2}(B)\right)=\left\langle\Omega, A \Delta^{-\frac{1}{2}} B \Omega\right\rangle$, where the modular operator $\Delta=e^{\pi K}$.

The first goal is to apply the KMS-condition to this matrix element, but to do this we must eliminate the $\Delta^{\frac{1}{2}}$ operator and rewrite in terms of operators from $\mathfrak{R}\left(X^{+}\right)$.

Using the theory of von Neumann algebras in standard form one can show, as in [BR, Proposition 2.5.26], that in $\mathscr{H}$ there is a self-dual positive cone $\mathscr{P}$ given by

$$
\overline{\Delta^{\frac{1}{4}} \mathfrak{R}\left(X^{+}\right)_{+} \Omega}=\overline{\Delta^{-\frac{1}{4}} \mathfrak{R}\left(X^{+}\right)_{+}^{\prime} \Omega}
$$

where $\mathfrak{R}_{+}$denotes the cone of positive elements in the von Neumann algebra $\mathfrak{R}$.

We first assume that $A, B \geq 0$ and that $B \in \mathfrak{R}\left(X^{+}\right)$is such that there is a $B^{\prime} \in \mathfrak{R}\left(X^{+}\right)^{\prime}, B^{\prime} \geq 0$, with the property $\Delta^{\frac{1}{4}} B^{\prime} \Omega=\Delta^{-\frac{1}{4}} B \Omega$.

The resolvent equation of [BR, Lemma 2.5.12] implies that there are operators $\tilde{B}, \hat{B} \in \mathfrak{R}\left(X^{+}\right)$such that

$$
\begin{aligned}
& (\Delta+1) \tilde{B}^{*} \Omega=B^{\prime} \Omega, \\
& (\Delta+1) \hat{B}^{*} \Omega=B \Omega .
\end{aligned}
$$


Before continuing, we remark that $\tilde{B}$ and $\hat{B}$ can be taken selfadjoint. This follows from the relation

$$
J B J=\Delta^{\frac{1}{2}} \hat{B} \Delta^{-\frac{1}{2}}+\Delta^{-\frac{1}{2}} \hat{B} \Delta^{\frac{1}{2}}
$$

on $\mathscr{D}\left(\Delta^{\frac{1}{2}}\right) \cap \mathscr{D}\left(\Delta^{-\frac{1}{2}}\right)$ [BR, Lemma 2.5.13]. Writing $\hat{B}=H+i K$ with $H, K$ selfadjoint we see from the positivity of $J B J$ that the form $\Delta^{\frac{1}{2}} K \Delta^{-\frac{1}{2}}+\Delta^{-\frac{1}{2}} K \Delta^{\frac{1}{2}}$ vanishes on $\mathscr{H}$. Thus $(\Delta+1) K \Omega=0$ and we can choose $K=0$ in (6). Similarly we can argue for $\tilde{B}$. Then this gives us

$$
\begin{aligned}
\left\langle\Omega, A \Delta^{\frac{1}{2}} B \Omega\right\rangle & =\left\langle\Omega, A B^{\prime} \Omega\right\rangle \\
& =\langle\Omega, A \tilde{B} \Omega\rangle+\langle\Omega, A \Delta \tilde{B} \Omega\rangle \\
& =\langle\Omega, A \tilde{B} \Omega\rangle+\left\langle\Delta^{\frac{1}{2}} A \Omega, \Delta^{\frac{1}{2}} \tilde{B} \Omega\right\rangle \\
& =\langle\Omega, A \tilde{B} \Omega\rangle+\frac{\left\langle J \Delta^{\frac{1}{2}} A \Omega, J \Delta^{\frac{1}{2}} \tilde{B} \Omega\right\rangle}{} \\
& =\langle\Omega, A \tilde{B} \Omega\rangle+\langle\Omega, A \tilde{B} \Omega\rangle .
\end{aligned}
$$

As $\tilde{B} \in \mathfrak{R}\left(X^{+}\right)$, we can apply the KMS-condition on both the terms. This gives us two complex functions, which add up to a $F$, analytic in the strip

$$
I=\{z \in \mathbb{C} \mid \operatorname{Im} z \in] 0,1[\}
$$

and bounded and continuous on $\bar{I}$, and satisfying for all real $t$

$$
F(t)=\left\langle\Omega, \tilde{B} \Delta^{-i t} A \Omega\right\rangle+\left\langle\Omega, A \Delta^{-i t} \tilde{B} \Omega\right\rangle
$$

and

$$
F(t+i)=\left\langle\Omega, \tilde{B} \Delta^{i t} A \Omega\right\rangle+\left\langle\Omega, A \Delta^{i t} \tilde{B} \Omega\right\rangle
$$

We see that $F$ satisfies $F(t)=F(-t+i)=\overline{F(-t)}$.

Claim. $F$ is real on the real axis: Consider the term

$$
\left\langle\Omega, \tilde{B} \Delta^{-i t} A \Omega\right\rangle=\left\langle\Omega, \tilde{B} A_{-t} \Omega\right\rangle
$$

where we put $A_{t}=\Delta^{i t} A \Delta^{-i t}$. Then $A_{-t}>0$ and we calculate

$$
\begin{aligned}
\left\langle\Omega, \tilde{B} A_{-t} \Omega\right\rangle & =\left\langle\tilde{B} \Omega, A_{-t} \Omega\right\rangle \\
& =\overline{\left\langle J \tilde{B} \Omega, \Delta^{\frac{1}{2}} A_{-t} \Omega\right\rangle} \\
& =\overline{\left\langle\Delta^{\frac{1}{4}} J \tilde{B} \Omega, \Delta^{\frac{1}{4}} A_{-t} \Omega\right\rangle} \\
& =\overline{\left\langle J \Delta^{-\frac{1}{4}}(\Delta+1)^{-1} B^{\prime} \Omega, \Delta^{\frac{1}{4}} A_{-t} \Omega\right\rangle} \\
& =\overline{\left\langle J \Delta^{\frac{1}{4}}(\Delta+1)^{-1} \hat{B} \Omega, \Delta^{\frac{1}{4}} A_{-t} \Omega\right\rangle} \\
& =\frac{\left\langle\Delta^{-\frac{1}{4}}(J \hat{B} J) \Omega, \Delta^{\frac{1}{4}} A_{-t} \Omega\right\rangle}{2} \geq 0 .
\end{aligned}
$$

In the last step we used again the self duality of the cone $\mathscr{P}$ which means exactly that the scalar product is positive (since $A_{-t} \in \mathfrak{R}\left(X^{+}\right)$and $\left.J \hat{B} J \in \mathfrak{R}\left(X^{+}\right)^{\prime}\right)$. Similarly one shows that the second term in $F(t)$ is real for any $t$.

Using Schwarz reflection, we extend $F$ analytically to $-I$ by $G(z)=\overline{F(\bar{z})}$, $z \in I$. We see that $G(t-i)=\overline{F(t+i)}=F(t+i)$. Thus $G(z+2 i)$ is an analytic function on $I+i$ which coincides with $F$ on the common boundary $\operatorname{Im} z=i$. 
Thus, by a simple version of the edge-of-the-wedge theorem, $G(z+2 i)$ is an analytic continuation of $F$. If we also denote by $F$ the new bounded analytic function on $2 I$, we see that the process can be repeated arbitrarily often to give us an entire bounded function, which is then constant by Liouville's theorem. Hence $F(0)=F(t)$ for all $t \in \mathbb{R}$. So

$$
\begin{aligned}
\left\langle\Omega, A \Delta^{-\frac{1}{2}} B \Omega\right\rangle & =\langle\Omega, \tilde{B} A \Omega\rangle+\langle\Omega, A \tilde{B} \Omega\rangle \\
& =\left\langle\Omega, \tilde{B} \Delta^{-i t} A \Omega\right\rangle+\left\langle\Omega, A \Delta^{-i t} \tilde{B} \Omega\right\rangle .
\end{aligned}
$$

Backtracing the steps with $A_{t} \in \mathfrak{R}\left(X^{+}\right)$substituted for $A$, this equality yields the following equation, which extends to all $A \in \mathfrak{R}\left(X^{+}\right)$by linearity:

$$
\left\langle A_{t} \Omega, \Delta^{-\frac{1}{2}} B \Omega\right\rangle=\left\langle A^{*} \Omega, \Delta^{-\frac{1}{2}} B \Omega\right\rangle .
$$

As $B$ is positive, the functional $\omega_{B}(\cdot)=\left\langle\Omega, \cdot \Delta^{-\frac{1}{2}} B \Omega\right\rangle /\left\langle\Omega, \Delta^{-\frac{1}{2}} B \Omega\right\rangle$ is linear, positive (this is proved like in (7)) and satisfies $\omega_{B}(1)=1$; hence it is a state on $\mathfrak{R}\left(X^{+}\right)$which is invariant under the modular group $\sigma_{t}$. By the hypothesis $\omega_{B}=\omega_{\Omega}$; and we obtain the conclusion

$$
\begin{aligned}
\left\langle\Omega, A \Delta^{-\frac{1}{2}} B \Omega\right\rangle & =\omega_{B}(A)\langle\Omega, B \Omega\rangle \\
& =\omega_{\Omega}(A)\langle\Omega, B \Omega\rangle \\
& =\langle\Omega, A \Omega\rangle\langle\Omega, B \Omega\rangle .
\end{aligned}
$$

Now consider the case where $B \geq 0$ is an arbitrary analytic element for $\sigma$. The mapping $C \mapsto \Delta^{-\frac{1}{4}} C \Omega$ is an order isomorphism from $\mathfrak{R}\left(X^{+}\right)_{+}^{\prime}$ with the ultra-weak topology to $\mathscr{P}$ with the weak topology [BR, Lemma 2.5.40]. We can find a net $\left(B_{\alpha}\right) \subset \mathfrak{R}\left(X^{+}\right)^{\prime}$ of positive, entire analytic elements for $\sigma$, which converges ultra-weakly to $B$, and each having the property that there exist a $B_{\alpha}^{\prime} \in \mathfrak{R}\left(X^{+}\right)_{+}$with $\Delta^{\frac{1}{4}} B_{\alpha}^{\prime} \Omega=\Delta^{-\frac{1}{4}} B_{\alpha} \Omega$. Thus we have the factorization (5) for each of the elements $B_{\alpha}$ and we obtain

$$
\begin{aligned}
\left\langle\Omega, A \Delta^{-\frac{1}{2}} B \Omega\right\rangle & =\left\langle\Delta^{-\frac{1}{4}} A \Omega, \Delta^{-\frac{1}{4}} B \Omega\right\rangle \\
& =\lim _{\alpha}\left\langle\Delta^{-\frac{1}{4}} A \Omega, \Delta^{-\frac{1}{4}} B_{\alpha} \Omega\right\rangle \\
& =\lim _{\alpha}\left\langle\Omega, A \Delta^{-\frac{1}{2}} B_{\alpha} \Omega\right\rangle \\
& =\lim _{\alpha}\langle\Omega, A \Omega\rangle\left\langle\Omega, B_{\alpha} \Omega\right\rangle \\
& =\langle\Omega, A \Omega\rangle\langle\Omega, B \Omega\rangle .
\end{aligned}
$$

Now noting that any $B \in \mathfrak{R}\left(X^{-}\right)$can be written in the form $B=\left(H_{1}-\right.$ $\left.H_{2}\right)+i\left(K_{1}-K_{2}\right)$ with $H_{1}, H_{2}, K_{1}$, and $K_{2}$ positive operators from $\mathfrak{R}\left(X^{-}\right)$, the above factorization of $\omega_{\Omega}$ readily extends to all $B \in \mathfrak{R}\left(X^{-}\right)$.

\section{A CONCRETE MODEL}

In this section we obtain a model satisfying the axioms (I)-(VI), the assumptions (3), (4), and ergodicity by certain modifications of the constructions in [D]. Thus this model will satisfy the conclusions of Theorems 3,4 , and 5 . 
We start by considering the classical Klein-Gordon equation $\left(\square+m^{2}\right) u=0$, where the d'Alembertian is $\square=\nabla^{\nu} \nabla_{\nu}$ and $m^{2}>0$ a mass-parameter. We notice that the Klein-Gordon equation is $L(\tau)$-covariant.

The solution theory of the Klein-Gordon equation on a globally hyperbolic manifold is well established and [D] gives an overview. There exist continuous fundamental solutions $E^{ \pm}: \mathscr{E}(X)^{\prime} \rightarrow \mathscr{D}^{\prime}(X)$ where $\mathscr{D}^{\prime}(X)$ denotes the space of distributions on $X$ and $\mathscr{E}^{\prime}(X)$ the space of distributions with compact support. We put $E=E^{+}-E^{-}$and have $E^{*}=-E$. Ef solves the KleinGordon equation for any $f \in C_{c}^{\infty}(X)$.

We can also give a description of the solution theory in terms of data on a given Cauchy surface $S \subset X$. Let $\rho_{0}: C^{\infty}(X) \rightarrow C^{\infty}(S)$ be the restriction mapping, and let $\rho_{1}: C^{\infty}(X) \rightarrow C^{\infty}(S)$ be the forward normal derivative in $S$. Then for any set of data $u_{0}, u_{1} \in C_{c}^{\infty}(S)$ there exists a unique solution $u \in C^{\infty}(X)$ with $\left(\square+m^{2}\right) u=0, \rho_{0}(u)=u_{0}$, and $\rho_{1}(u)=u_{1}$. If $\rho_{0}^{\prime}, \rho_{1}^{\prime}$ : $\mathscr{D}^{\prime}(S) \rightarrow \mathscr{D}^{\prime}(X)$ denote the adjoints of $\rho_{0}$ and $\rho_{1}$ respectively, then $E \rho_{0}^{\prime}$ and $E \rho_{1}^{\prime}$ restrict continuously to operators from $C_{c}^{\infty}(S)$ to $C^{\infty}(X)$, and the difference

$$
u=E \rho_{0}^{\prime} u_{1}-E \rho_{1}^{\prime} u_{0}
$$

is the solution to the Cauchy problem with data $\left(u_{0}, u_{1}\right)$. The connection between the two formulations is provided by the lemma

Lemma 6 [D]. Let $u$ be a solution to $\left(\square+m^{2}\right) u=0$, and let $\rho_{0}(u)$ and $\rho_{1}(u)$ have support in a compact subset $N \subset S$. Then, for any open neighborhood $\mathscr{O}$ of $N$, there exists $f \in C_{c}^{\infty}(X)$ such that supp $f \subset \mathcal{O}$ and $u=E f$.

Consider the Cauchy surface $S=\{x=(t, w, y) \in X \mid t=0\}$, and put $S^{ \pm}=$ $\left\{x=(t, w, y) \in X \mid w \in \mathbb{R}_{ \pm}\right\}$. We introduce an involution $\mathfrak{J}$ on $C^{\infty}(X)$ by $(\mathfrak{J} f)(t, w, y)=i f(t,-w, y)$. $\mathfrak{J}$ restricts to an isomorphism between $C_{c}^{\infty}\left(S^{+}\right)$ and $C_{c}^{\infty}\left(S^{-}\right)$. The symmetry $w \mapsto-w$ of $X$ implies that $\mathfrak{J}$ commutes with $\rho_{0}, \rho_{1}$, and $E$. Using $\mathfrak{J}$ we set

$$
\sigma\left(\left(h_{1}, h_{1}^{\prime}\right),\left(h_{2}, h_{2}^{\prime}\right)\right)=\left\langle h_{1}, \mathfrak{J} h_{2}^{\prime}\right\rangle-\left\langle h_{2}, \mathfrak{J} h_{1}^{\prime}\right\rangle
$$

to obtain a nondegenerate symplectic form on $C_{c}^{\infty}(S) \oplus C_{c}^{\infty}(S)$.

We construct the net of local observables by specifying a representation of the symplectic space $\left(C_{c}^{\infty} \oplus C_{c}^{\infty}(X), \sigma\right)$ on the standard Fock space. Let us start by describing this.

We let $\mathscr{H}_{0}=\mathbb{C}$ and let $\mathscr{H}_{1}$ denote the completion of $C_{c}^{\infty}(S)$ with respect to the inner product

$$
\left(h, h^{\prime}\right)=\int h \bar{h}^{\prime} d S
$$

Let $\mathscr{H}_{n}=\bigotimes_{\text {sym }}^{n} \mathscr{H}_{1}$ be the symmetric tensor product of $n$ copies of $\mathscr{H}_{1}$, and let $\mathscr{H}=\bar{\bigoplus}_{n=0}^{\infty} \mathscr{H}_{n}$ be the symmetrized Fock space. Using the annihilation operators $a(h)$ and the creation operators $a(h)^{*}$, we define the symmetric operators

$$
\begin{aligned}
& \theta(h)=\left(a^{*}(h)+a(h)\right) / \sqrt{2}, \\
& \pi(h)=i\left(a^{*}(h)-a(h)\right) / \sqrt{2}
\end{aligned}
$$

which then satisfy $\left[\theta(h), \pi\left(h^{\prime}\right)\right]=\left(h, \bar{h}^{\prime}\right)$. Putting

$$
W\left(h, h^{\prime}\right)=e^{i\left(\theta(h)-\pi\left(\mathfrak{J} h^{\prime}\right)\right)}
$$


we obtain a representation of the canonical commutator relations over $S$. W is a twisting of the Fock representation by the involution $\mathfrak{J}$ and satisfies the Weyl relation

$$
W\left(h_{1}, h_{1}^{\prime}\right) W\left(h_{2}, h_{2}^{\prime}\right)=W\left(h_{1}+h_{2}, h_{1}^{\prime}+h_{2}^{\prime}\right) e^{-i \sigma\left(\left(h_{1}, h_{1}^{\prime}\right),\left(h_{2}, h_{2}^{\prime}\right)\right) / 2} .
$$

We define the net of local observables by setting

$$
\mathfrak{R}(\mathscr{O})=\left\{W\left(\rho_{1} E f, \rho_{0} E \mathfrak{J} f\right) \mid f \in C_{c}^{\infty}(X, \mathbb{R}), \operatorname{supp} f \subset \mathscr{O}\right\}^{\prime \prime}
$$

for a region $\mathscr{O} \subset X$. Here $C_{c}^{\infty}(X, \mathbb{R})$ denotes the real subspace of real-valued functions in $C_{c}^{\infty}(X)$.

The wedge-algebras $\mathfrak{R}\left(X^{+}\right)$(resp. $\mathfrak{R}\left(X^{-}\right)$) can be described as the von Neumann algebra generated by the $W\left(h, \mathfrak{J} h^{\prime}\right)$ with $h, h^{\prime} \in C_{c}^{\infty}\left(S^{+}, \mathbb{R}\right)$ (resp. $\left.C_{c}^{\infty}\left(S^{-}, \mathbb{R}\right)\right)$. This is a consequence of Lemma 6 , from which we conclude that for $\left(h, h^{\prime}\right) \in C_{c}^{\infty}\left(S^{+}\right) \oplus C_{c}^{\infty}\left(S^{+}\right)$, we can choose a function $f$ with $\left(E \rho_{1} f, E \rho_{0} \mathfrak{J} f\right)=\left(h, \mathfrak{J} h^{\prime}\right)$, such that $f$ is supported in $X^{+}$.

We then obtain the main theorem of this section.

Theorem 7. The net $\mathscr{O} \rightarrow \mathfrak{R}(\mathscr{O})$ satisfies the axioms $(\mathrm{I})-(\mathrm{VI})$, the assumptions (3) and (4), and ergodicity.

Proof. Axiom (I) is a simple consequence of the support properties of functions.

To show axiom (II), let a region $\mathscr{O} \subset X$ satisfy $\mathscr{O}=\bigcup_{i \in J} \mathscr{O}_{i}, \mathscr{O}_{i} \in B(X)$. From axiom (I), we get the inclusion $\left(\bigcup_{i \in J} \mathfrak{R}\left(\mathscr{O}_{i}\right)\right)^{\prime \prime} \subset \mathfrak{R}(\mathscr{O})$. To see the other inclusion, let $f \in C_{c}^{\infty}(X)$, with supp $f \subset \mathscr{O}$. As supp $f$ is compact, we can choose a finite subcovering $\left\{\mathscr{O}_{i_{k}}\right\}$ of supp $f$ from $\left\{O_{i}\right\}$. Let $\left\{h_{i_{k}}\right\}$ be a smooth partition of unity, subordinate to the covering $\left\{\mathscr{O}_{i_{k}}\right\}$. Then $f=\sum h_{i_{k}} f$. Using (10) we get

$$
\begin{aligned}
& W\left(\rho_{1} E f, \rho_{0} E \mathfrak{J} f\right)=W\left(\sum \rho_{1} E\left(h_{i_{k}} f\right), \sum \rho_{0} E\left(h_{i_{k}} \mathfrak{J} f\right)\right) \\
& \quad=k W\left(\rho_{1} E\left(h_{1} f\right), \rho_{0} E\left(h_{1} \mathfrak{J} f\right)\right) \cdots W\left(\rho_{1} E\left(h_{i_{k}} f\right), \rho_{0} E\left(h_{i_{k}} \mathfrak{J} f\right)\right)
\end{aligned}
$$

where $k$ is a complex number. From this it follows that $\mathfrak{R}(\mathscr{O}) \subset\left(\bigcup_{i \in J} \mathfrak{R}\left(\mathscr{O}_{i}\right)\right)^{\prime \prime}$.

To see that the time-slice axiom (III) is fulfilled we notice that the global algebra is generated by the unitaries $W\left(h, h^{\prime}\right)$, with $h, h^{\prime} \in C_{0}^{\infty}(S)$. Now apply Lemma 6.

For the locality axiom (IV), we first remark that (8) implies

$$
\begin{aligned}
\left\langle\rho_{1} E\right. & \left.f_{1}, \rho_{0} E f_{2}\right\rangle-\left\langle\rho_{1} E f_{2}, \rho_{0} E f_{1}\right\rangle=\left\langle f_{1},-E \rho_{1}{ }^{\prime} \rho_{0} E f_{2}\right\rangle-\left\langle-E \rho_{0}{ }^{\prime} \rho_{1} E f_{2}, f_{1}\right\rangle \\
& =\left\langle f_{1},\left(E-E \rho_{0}{ }^{\prime} \rho_{1} E\right) f_{2}\right\rangle+\left\langle E \rho_{0}{ }^{\prime} \rho_{1} E f_{2}, f_{1}\right\rangle \\
& =\left\langle f_{1}, E f_{2}\right\rangle+2 \operatorname{Re}\left\langle f_{1}, E \rho_{0}{ }^{\prime} \rho_{1} E f_{2}\right\rangle
\end{aligned}
$$

This equation together with the one with $f_{1}$ and $f_{2}$ interchanged and (10) gives

$$
\begin{aligned}
& W\left(\rho_{1} E f_{1}, \rho_{0} E \mathfrak{J} f_{1}\right) W\left(\rho_{1} E f_{2}, \rho_{0} E \mathfrak{J} f_{2}\right) \\
& \quad=W\left(\rho_{1} E f_{2}, \rho_{0} E \mathfrak{J} f_{2}\right) W\left(\rho_{1} E f_{1}, \rho_{0} E \mathfrak{J} f_{1}\right) e^{i\left\langle f_{1}, E f_{2}\right\rangle} .
\end{aligned}
$$

If $\mathscr{O}_{1}$ and $\mathscr{O}_{2}$ are given spacelike separated regions with supp $f_{1} \subset O_{1}$ and supp $f_{2} \subset \mathscr{O}_{2}$, then the two supports cannot be joined by a causal curve. By the causal propagation properties of $E$ we have $\left\langle f_{1}, E f_{2}\right\rangle=0$, so that

$$
\left[W\left(\rho_{1} E f_{1}, \rho_{0} E \mathfrak{J} f_{1}\right), W\left(\rho_{1} E f_{2}, \rho_{0} E \mathfrak{J} f_{2}\right)\right]=0 .
$$


As these unitaries generate $\mathfrak{R}\left(\mathscr{O}_{1}\right)$ and $\mathfrak{R}\left(\mathscr{O}_{2}\right)$ respectively, we get $\mathfrak{R}\left(\mathscr{O}_{1}\right) \subset$ $\mathfrak{R}\left(\mathscr{O}_{2}\right)^{\prime}$ proving axiom (IV).

To show the $L(\tau)$ covariance of axiom (V), we first remark that we have automorphisms $L(\tau)^{*}: C_{c}^{\infty}\left(X^{+} \cup X^{-}\right) \rightarrow C_{c}^{\infty}\left(X^{+} \cup X^{-}\right)$given by $\left(L(\tau)^{*} f\right)(x)$ $=f(L(\tau) x)$. Furthermore, since the Klein-Gordon equation on $X$ is $L(\tau)$ covariant, it easily follows that

$$
L(\tau)^{*} E \rho_{i} f=E \rho_{i} L(\tau) f \text { for all } f \in C_{0}^{\infty}(X) \text { and } i=0,1
$$

Thus $\mathrm{L}(\tau)^{*}$ induce Bogoljubov transformations $\tilde{L}(\tau)$ on $\mathfrak{R}\left(X^{+} \cup X^{-}\right)$by

$$
\tilde{L}(\tau) W\left(\rho_{1} E f, \rho_{0} E \mathfrak{J} f\right)=W\left(\rho_{1} E L(\tau)^{*} f, \rho_{0} E L(\tau)^{*} \mathfrak{J} f\right) .
$$

But standard second quantization techniques then yield that $\tilde{L}(\tau)$ is strongly continuous and is unitarily implemented on $\overline{\mathfrak{R}\left(X^{+} \cup X^{-}\right) \Omega}$ by the unitaries $L(\tau)$ defined by

$$
L(\tau)(A \Omega)=(\tilde{L}(\tau) A) \Omega \text { for } A \in \mathfrak{R}\left(X^{+} \cup X^{-}\right) .
$$

As $C_{c}^{\infty}\left(S^{+} \cup S^{-}\right)$is dense in $C_{c}^{\infty}(S)$ in the norm given by (9), $\Omega$ is a cyclic vector for $\mathfrak{R}\left(X^{+} \cup X^{-}\right)$. Thus the definition above yields a strongly continuous unitary group on all of $\mathscr{H}$.

To show axiom (VI), we remark that the real linear subspace

$$
\mathscr{K}=C_{c}^{\infty}\left(S^{+}, \mathbb{R}\right) \oplus i C_{c}^{\infty}\left(S^{-}, \mathbb{R}\right)
$$

of $\mathscr{H}_{1}$ has the property that $\mathscr{K}+i \mathscr{K}$ is dense in $\mathscr{H}_{1}$. Thus by a theorem of Araki [A], $\Omega$ is cyclic for the von Neumann algebra generated by the CCR over $\mathscr{K}$. But this algebra is exactly $\mathfrak{R}\left(X^{+}\right)$as remarked after the definition of the local algebras. Since a similar argument yields that $\Omega$ is cyclic for $\mathfrak{R}\left(X^{-}\right)$, we have verified axiom (VI). In fact [A] gives us more. Since $\overline{\mathscr{K}} \cap i \overline{\mathscr{K}}=\{0\}$, the duality (1) is fulfilled, and thus also (4) (and by locality (3)). So $L(2 \pi i)$ is the modular operator for $\left(\mathfrak{R}\left(X^{+}\right), \Omega\right)$ by Theorem 3 .

We finally show that $\mathbb{C}$ and $\Omega$ are precisely the $L(\tau)$-invariant vectors in $\mathscr{H}$. As remarked in the beginning of section II we can always take a normal state on $\mathfrak{R}\left(X^{+}\right)$to be implemented by a unit vector when there exist bicyclic vectors for $\mathfrak{R}\left(X^{+}\right)$. Thus this is equivalent to the ergodicity hypothesis.

Assume first that for all $\tau \in \mathbb{R}, L(\tau)^{*} g_{i}=g_{i}$ for $g_{i} \in C_{0}^{\infty}(S), i=0,1$. By Lemma 6, there exist a $f \in C_{c}^{\infty}(X)$ such that $u=E f$ solves the Klein-Gordon equation and $g_{i}=\rho_{i}(u)$. Thus $\rho_{i} E L(\tau)^{*} f=\rho_{i} E f$ or $\rho_{i}\left(u-L(\tau)^{*} u\right)=0$. As the Klein-Gordon equation is covariant, $u-L(\tau)^{*} u$ solves it, and we get from uniqueness of solutions that $u=L(\tau)^{*} u$ for all $\tau \in \mathbb{R}$. Thus $u$ is independent of $\tau$ and $\tilde{u}=\left.u\right|_{s}$ solves the reduced wave equation $\left(\Delta_{S}+m^{2}\right) \tilde{u}=0$ on $S$. As $\tilde{u}$ is smooth on $S, V=\operatorname{supp} \tilde{u}$ has smooth boundary. So $\tilde{u}$ solves the Dirichlet problem $\left(\Delta_{S}+m^{2}\right) u=0$ on $S, \tilde{u}=0, \nabla \tilde{u}=0$ on $\partial V$. This implies that $\tilde{u}=0$ (this uniqueness theorem follows, e.g., from the variational techniques in [KS]). As $u$ is $\tau$ independent, $u=0$ and $g_{0}=g_{1}=0$. So no nonzero vector in $h_{1}$ is invariant under $L(\tau)^{*}$, and we conclude as wanted that $\mathbb{C} \Omega$ is the subspace of invariant vectors for $L(\tau)$. 


\section{REFERENCES}

[A] H. Araki, A lattice of von Neumann algebras associated with the quantum theory of a free Bose field, J. Math. Phys. 4 (1963), 1343-1362.

[BR] O. Bratteli and D. W. Robinson, Operator algebras and quantum statistical mechanics 1-2, Springer, New York, 1967.

[BW] J. J. Bisognano and E. H. Wichmann, On the duality condition for a Hermitean scalar field, J. Math. Phys. 12 (1975), 985-1007.

[D] J. Dimock, Algebras of local observables on a manifold, Comm. Math. Phys. 77 (1980), 219-228.

[Da] P. C. W. Davies, Scalar particle production in Schwartzscild and Rindler metrics, J. Phys. A 8 (1975), 609-617.

[F] K. Fredenhagen, On the modular structure of local algebras of observables, Comm. Math. Phys. 97 (1985), 79-90.

[Fu] S. A. Fulling, Alternative vacuum states in static spacetimes with horizons, J. Phys. A 10 (1977), 917-951.

[H] R. Haag, Local quantum physics, Springer, New York, 1992.

[Ha] S. W. Hawking, Particle creation by black holes, Comm. Math. Phys. 43 (1975), 199-220.

[HE] S. W. Hawking and G. E. R. Ellis, The large-scale structure of spacetime, Cambridge University Press, London, 1973.

[HHW] R. Haag, N. M. Hugenholtz and M. Winnink, On the equilibrium states in quantum statistical mechanics, Comm. Math. Phys. 5 (1967), 215-236.

[HK] R. Haag and D. Kastler, An algebraic approach to quantum field theory, J. Math. Phys. 5 (1964), 848-861.

[Ho] S. S. Horozhy, Introduction to algebraic quantum field theory, Kluwer, Holland, 1990.

[Ka] B. S. Kay, The double-wedge algebra for quantum fields on Schwartzschild and Minkowski space-times, Comm. Math. Phys. 100 (1985), 57-91.

[KR] R. V. Kadison and J. R. Ringrose, Fundamentals of the theory of operator algebras. I, II, Academic Press, 1986.

[KS] D. Kinderlehrer and G. Stampacchia, An introduction to variational inequalities and their applications, Academic Press, 1980.

[O] M. Olesen, A type III $_{\lambda}$ field theory, Preprint 1993.

[PT] G. K. Pedersen and M. Takesaki, The Radon-Nikodym theorem for von Neumann algebras, Acta Math. 130 (1973), 53-87.

[R] C. Rigotti, Remarks on the modular operator and local observables, Comm. Math. Phys. 37 (1974), 273-286.

[RvD] M. A. Rieffel and A. van Daele, The commutation theorem for tensor products of von Neumann algebras, London Math. Soc. 7 (1975), 257-260.

[Se] G. L. Sewell, Quantum fields on manifolds: PCT and gravitationally induced thermal states, Ann. Phys. 141 (1982), 201-224.

[St] E. Størmer, Types of von Neumann algebras associated with extremal invariant states, Comm. Math. Phys. 6 (1967), 194-204.

[U] W. G. Unruh, Notes on black hole evaporation, Phys. Rev. D 14 (1976), 870-892.

Department of Mathematics, University of Iowa, Iowa City, Iowa 52242

E-mail address: olesene math.uiowa.edu

Matematisk Institut, University of Copenhagen, Universitetsparken 5, DK-2100 Copenhagen, Denmark

E-mail address: olesene euromath.dk 